\title{
AlGaAs TO GaAs ENERGY TRANSFER MECHANISMS IN AlGaAs/GaAs STRUCTURES*
}

\author{
K. KaRPińsKa, M. Godlewski, Z.R. ŻYTKIEwICZ \\ Institute of Physics, Polish Academy of Sciences \\ Al. Lotników 32/46, 02-668 Warszawa, Poland
}

W.M. Chen AND E.R. Weber

Dept. of Materials Science and Mineral Engineering, University of California Berkeley, CA 94720, USA

The results of photoluminescence and optically detected cyclotron resonance experiments are presented for thick AlGaAs epilayers grown by liquid phase electroepitaxy method on GaAs:Cr substrate. These results indicate an efficient energy transfer from excited AlGaAs to GaAs.

PACS numbers: $72.20 . \mathrm{Jv}, 76.70 . \mathrm{Hb}, 78.55 . \mathrm{Cr}$

\section{Introduction}

Intensive studies of AlGaAs-GaAs heterostructures were stimulated by the high quantum efficiency of the resulting opto-electronic devices [1]. It was reported previously that coating with a thin layer of AlGaAs may enhance the GaAs photoluminescence (PL). One of the reasons for such enhancement is the reduced probability of nonradiative recombination at the interface as compared to that at the GaAs surface [1]. Recently it was reported that a bright GaAs emission can be observed for the above-band-gap excitation of AlGaAs in AlGaAs-GaAs heterostructures [2]. The relevant mechanism of energy transfer remained, however, unknown. In this paper we present new experimental results of PL and of optically detected cyclotron resonance (ODCR) which allow us to verify the nature of this mechanism.

*This work was partly supported by the grant no. 204769101 of the Committee for Scientific Research. 


\section{Experimental}

Direct band gap AlGaAs epilayers were grown by liquid phase electroepitaxy (LPEE) method on (100) GaAs:Cr substrates. The LPEE method allows to control the $\mathrm{Al}$ concentration distribution during the growth. This enabled us to obtain samples with $\mathrm{Al}$ mole fraction increasing not only towards the substrate, as common for LPE, but also towards the surface [3]. This was the crucial point in the present study and helped us to verify the mechanisms of energy transfer from epilayer to substrate. Epilayers were doped with Te to the level of $10^{17} \mathrm{~cm}^{-3}$. Two different samples were used. The first (E5/92) was a $55 \mu \mathrm{m}$ thick epilaycr with $\mathrm{Al}$ mole fraction changing linearly from 0.225 at surface to 0.20 at interface. The second (E24/91) was a $20 \mu \mathrm{m}$ thick epilayer with $\mathrm{Al}$ mole fraction changing linearly from 0.15 at surface to 0.25 at interface.

\section{Results and discussion}

In Fig. 1 we show the PL spectrum of sample E5/92 studied under the above-band-gap AlGaAs excitation. The PL spectrum observed consists of the

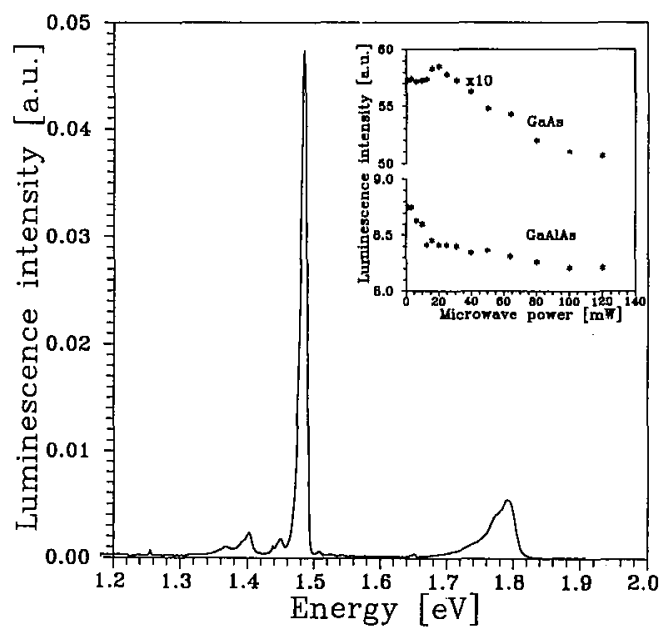

Fig. 1. The photoluminescence spectrum of the AlGaAs-GaAs heterostructure under the $514.5 \mathrm{~nm}$ excitation on AlGaAs-side. In the inset the influence of the microwave power on PL intensity of AlGaAs and GaAs (C-related DAP) emissions is presented.

AlGaAs donor-acceptor pair (DAP) emission at $6920 \mathrm{~nm}(1.791 \mathrm{eV})$ [4] and GaAs emissions with $\mathrm{C}_{\mathrm{As}}$-related DAP PL at $1.493 \mathrm{eV}$ [5], Mn $\mathrm{Ma}_{\mathrm{G}}$-related DAP PL at $1.403 \mathrm{eV}[6]$ and an unidentified deep DAP PL at about $1 \mathrm{eV}$. It is interesting to notice that the $1.791 \mathrm{eV}$ PL fits to DAP emission of AlGaAs with Al mole fraction of about 0.2 and is due to the Te donor-C acceptor transition [4]. In Fig. 2 (a,b) we show the edge GaAs PL of two samples studied under the AlGaAs- and GaAs-side excitation. Much brighter GaAs emission is observed under AlGaAs-side excitation for the sample E5/92. 


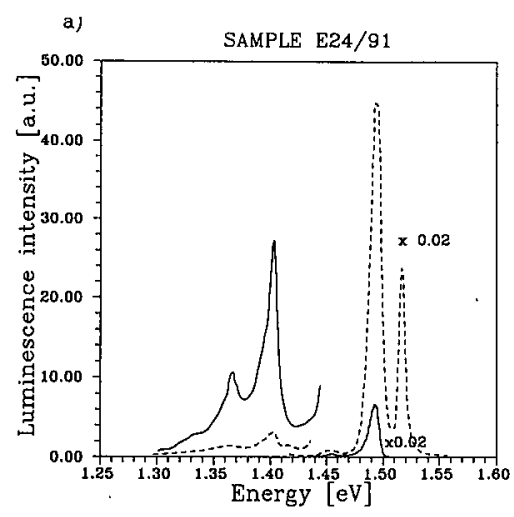

b)

SAMPLE E5/92

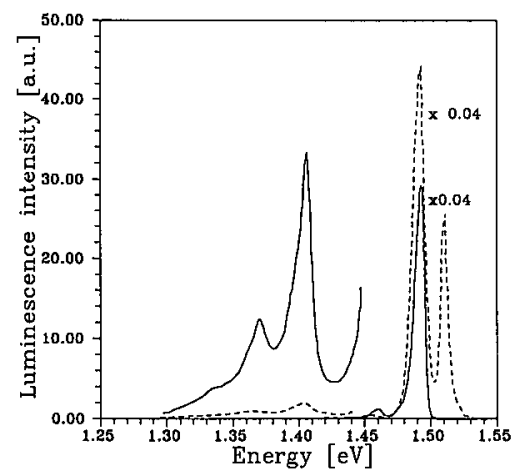

Fig. 2. The PL spectra of the emission of the GaAs substrate under the $488 \mathrm{~nm}$ excitation on $\mathrm{AlGaAs}$-side (solid line) and GaAs-side (dashed line). The results for two different samples are presented which differ by slope of the band gap energies towards the interface.

Two possible AlGaAs to GaAs energy transfer mechanisms were proposed previously, i.e. the radiative energy transfer and carrier migration from excited $\mathrm{AlGaAs}$ to $\mathrm{GaAs}[2,7]$. For a sample with $\mathrm{Al}$ fraction increasing towards the interface, i.e. with the band gap of AlGaAs increasing towards the interface, radiative energy transfer from AlGaAs to GaAs should be more probable due to reduced light reabsorption. For a sample with $\mathrm{Al}$ mole fraction decreasing towards the interface, light emitted at the AlGaAs surface should be reabsorbed already within the AlGaAs epilayer before it can reach the interface. This will stimulate the AlGaAs emission of smaller Al mole fraction, as observed in the experiment, and possibly increase the chance of free carrier migration from AlGaAs to GaAs.

In the inset of Fig. 1 we show the results of the ODCR experiment which was performed to verify the probability of carrier migration from AlGaAs to $\mathrm{GaAs}$ in the sample E5/92. It was shown previously that impact ionization of shallow donors in AlGaAs may enhance free carriers migration to GaAs [8]. A similar re- 
sult is obtained in our experiment. Impact ionization of the shallow AlGaAs donor (about $6 \mathrm{meV}$ ionization energy [4]) deactivates the AlGaAs DAP emission which results in an enhancement of GaAs DAP PL. This observation indicates that carrier migration between $\mathrm{AlGaAs}$ and GaAs may take place and most likely accounts for the enhanced brightness of the GaAs emission in the sample E5/92. The radiative energy transfer is seemingly less efficient. AlGaAs emission is reabsorbed at the AlGaAs-GaAs interface which may result in an increased probability of nonradiative transitions due to increased lattice defect concentration the heterojunction region.

An interesting question to be answered is the relation between carrier migration probability and the slope of the AlGaAs band gap towards the interface. We expect that carrier migration for the sample E5/92 is enhanced by the decreasing $\mathrm{Al}$ mole fraction towards the interface. This slope causes that free carriers generated with light at the AlGaAs surface can be swept towards the interface. This also will reduce the probability of recombination at the surface, i.e. in the region of enhanced nonradiative transitions.

\section{References}

[1] M.B. Panish, Proceedings of the IEEE 64, 1512 (1976).

[2] M. Godlewski, K. Fronc, W.M. Chen, B. Monemar, Acta Phys. Pol. A 80, 341 (1991).

[3] Z.R. Żytkiewicz, S. Miotkowska, this conference.

[4] D.J. Ashen, P.J. Dean, P.D. Greene, D.T.J. Hurle, J.B. Mullin, A.M. White, J. Phys. Chem. Solids 36, 1041 (1975); R. Dingle, R.A. Logan, J.R. Arthur, Inst. Phys. Conf. Ser. No. 33a, 210 (1977).

[5] Y.R. Yuan, M.A.A. Pudensi, G.A. Vawter, J.L. Merz, J. Appl. Phys. 58, 397 (1985).

[6] W. Schairer, M. Schmidt, Phys. Rev. B 10, 2501 (1974).

[7] R.J. Roedel, V.G. Karamandas, J. Appl. Phys. 50, 6353 (1979).

[8] W.M. Chen, P.O. Holtz, B. Monemar, M.Sundaram, J.L. Merz, A.C. Gossard, Proc. MRS Fall Meeting, Boston 1989. 